\title{
Mayalanmış Ev Yoğurdunun Helikobakter Pilori Eradikasyonuna Etkisi
}

\section{The Effect of Fermented Homemade-Style Yogurt in Helicobacter Pylori Eradication}

\author{
Şengül Aydın Yoldemir, ${ }^{1}$ Gülden Yürüyen²
}

\section{ÖZET}

Amaç: Helikobakter pilori $(\mathrm{Hp})$ kronik gastrit, peptik ülser ve gastrik malignitenin patogenezinde yer alan tüm dünyada yaygın olarak görülen bir enfeksiyondur. Kullanılan tedavilerin etkinliği ve Hp 'nin antibiyotik direnci nedeni ile yeni arayışlar gündeme gelmiştir. Tedaviye probiyotik içeren yoğurt ilavesinin Hp eradikasyon oranlarını anlamlı derecede arttırdığını gösteren çalışmalar vardır. Bu çalışmada birinci basamak standart 3'lü tedaviye evde mayalanmış taze yoğurt eklenmesinin Hp eradikasyon hızına ve hasta uyumu üzerine etkisi araştırdık.

Yöntem: Çalışmaya dispepsi sebebiyle üst gastrointestinal endoskopisi yapılan 147 hasta alındı. Endoskopik incelemede histolojik yöntemle Hp enfeksiyonu teşhis edilen hastalara eradikasyon tedavisi verildi. Hastaların evde mayalanmış yoğurt tüketimi sorgulandı. Reçete edilen ilaçların \%80'inden fazlasını alan hastalar tedaviye uyumlu kabul edildi. Tedavi bitiminden 4 hafta sonra, Hp eradikasyonu gaitada $\mathrm{Hp}$ antijen testi ile değerlendirildi.

Bulgular: ITT analizde yoğurt+ 3'lü tedavi alan 87 hastanın 50'sinde(\%57.4), sadece 3'lü tedavi alan 60 hastanın $21^{\prime}$ inde(\%39) eradikasyon sağlandı. PP analizde yoğurt+ 3'lü tedavi alan 65 hastanın 50'inde (\%76.9), sadece 3'lü tedavi alan 40 hastanın 21 'inde (\%52.5) Hp eradikasyonu sağlandı. Yoğurt+3'lü tedavi alanlarda daha iyi eradikasyon oranları elde edildi $(p=0.009 ; p=0.028)$.

Sonuç: Standart 3'lü tedaviye eklenen geleneksel ev yoğurdu ile Hp eradikasyon hızı artırmıştır. Bununla birlikte, yaş cinsiyet, eğitim düzeyi gibi faktörler eradikasyonu etkilememiştir.

Anahtar sözcükler: Helikobakter; eradikasyon; yoğurt.

\section{ABSTRACT}

Objectives: Helicobacter pylori ( $\mathrm{Hp}$ ) infection is common worldwide and may be involved in the pathogenesis of gastritis, peptic ulcer, and gastric malignancy. Treatment has been effective; however, due to the increasing antibiotic resistance of $\mathrm{Hp}$, there is renewed interest in research. Studies have indicated that the addition of probiotic yogurt to treatment may increase the rate of $\mathrm{Hp}$ eradication. This study was an investigation of the effect of the addition yogurt to the standard, first-line treatment on $\mathrm{Hp}$ eradication and patient compliance.

Methods: A total of 147 patients were included in the study. Patients who were diagnosed with Hp infection in an endoscopic examination using the histological method were given eradication treatment. The consumption of yogurt in the home was questioned. Patients who used at least $80 \%$ of the prescribed drugs were considered to have complied with the treatment. A stool antigen test was performed 4 weeks after the end of treatment to assess $\mathrm{Hp}$ eradication.

Results: Intention-to-treat analysis revealed that eradication was achieved in 50 (57.4\%) of the 80 patients who received only first-line treatment and 21 (39\%) of the 60 patients receiving yogurt + first-line treatment. Per-protocol analysis indicated that $\mathrm{Hp}$ eradication was achieved in 50 patients (76.9\%) of the 65 patients who received yogurt + first-line treatment. Yogurt + first-line treatment had a better eradication rate $(p=0.009 ; p=0.028)$.

Conclusion: The Hp eradication rate increased with the addition of yogurt added to the standard treatment. Factors such as age, gender, and education level did not affect eradication.

Keywords: Helicobacter; eradication; yogurt. 
$\mathrm{H}$ elikobakter pilori (Hp) tüm dünyada yaygın olarak görülen bir enfeksiyondur. ${ }^{[1-3]}$ Hp'nin 1983'te keşfinden bu yana kronik gastrit, peptik ülser ve gastrik malignitenin patogenezinde önemli bir rol oynadığını gösteren bir çok çalışma yapılmıştır. Gelişmekte olan ülkeler başta olmak üzere dünya nüfusunun yaklaşık\% 50'sinin Hp ile enfekte olduğu düşünülmektedir. [4]

HP eradikasyon şu anda standart tedavidir ve kronik gastrit, peptik ülser rekürrensi ve malign değişimi önleyebilir. [5] Hp'nin yok edilmesi, hala geçerli olan ve birinci ve ikinci tedavi yöntemleri sırasıyla proton pompa inhibitörü (PPI) + klaritromisin + amoksisilin/metronidazol veya bizmut + PPI + tetrasiklin + metronidazol dahil üçlü ve dörtlü tedavi rejimlerine dayanmaktadır sırasıyla. [6]

En yaygın kullanılan tedavilerin etkinliği, Hp'nin antibiyotik dirençli suşlarının hızla ortaya çıkması ve hastaların tedaviye uyumunun zayıf olmasıyla giderek daha fazla tehlikeye girmektedir. ${ }^{[7]}$ Bununla birlikte, klaritromisin direncinin düşük olduğu alanlarda veya tedavi ön tedavi yatkınlığı testine dayalı olarak seçildiğinde, üçlü tedavi halen tavsiye edilmektedir.

Ortaya çıkan bu durum nedeniyle yeni arayışlar gündeme gelmiştir. Yıllardan beri PPI'lı üçlü tedavilerin etkinliğini arttırmak için birlikte probiyotik kullanımı çalışmaları yapilmaktadır.

Probiyotiklerle takviyenin Hp eradikasyon oranlarını iyileştirip iyileştirmediği konusunda bazı tartışmalar olsada, birkaç meta-analiz ve inceleme makalesi, probiyotiklerin Hp eradikasyon oranını yaklaşık \%5-10 oranında artırabildiğini öne sürmektedir. [8-11]

Yoğurt laktik asit üreten birçok bakterinin (Lactobacillus acidophilus, Lactobacillus bulgaricus, Streptcus thermophilicus vs.) sütü fermente etmesiyle oluşur. Yoğurt üretiminde geleneksel olarak sütteki laktozu, laktik aside çeviren iki bakteri, Streptococcus salivarius subsp. thermophilus ve Lactobacillus delbrueckii subsp. Bulgaricus işbirliği içindedir. ${ }^{[12]}$

Yoğurt bakterilerinin probiyotik özellikte oldukları ve pH'1 düşürerek aside duyarlı bakterilerin üremelerini inhibe ettikleri bilinmektedir. Laktik asit bakterilerinin ürettiği antimikrobiyal maddeler patojen ve saprofit çeşitli bakterilerin gelişmesini inhibe ederler. Bu maddeler başta laktik asit olmak üzere; asetik asit, formik asit, fenil laktik asit, kaproik asit, organik asitler, etanol, hidrojen peroksit, diasetil, bakteriyosinler ve bakterisidal proteinlerdir. ${ }^{[13]} \mathrm{Bu}$ maddelerin aktivitesi yardımı ile özellikle Clostridium difficile ve Helicobacter pylori gibi patojen mikroorganizmaların neden olduğu gastrointestinal sistem enfeksiyonlarının tedavisin- de ve bu patojenlerin kolonizasyonlarının engellenmesinde de önemli rol oynamaktadırlar. ${ }^{[14]}$ Yoğurt bakterilerinden laktobasillus supp Hp'ye karşı hem in vitro hemde in vivo olarak antimikrobiyal aktivite göstermiştir. ${ }^{[15,16]}$

Yapılan bazı çalışmalarda üçlü tedaviye probiyotik içeren yoğurt ilavesinin Hp eradikasyon oranlarını anlamlı derecede arttırdığını göstermiştir. ${ }^{[17,18]}$ Randomize olmayan bir çalışmada fermente süt ürünlerinin günde $2 \mathrm{kez}$ tüketilmesi ile 8 haftalık takip sonrasında Hp kolonizasyonunu baskılayabileceği sonucuna varılmıştır. $[17,18]$

Ülkemizde probiyotik içeren yoğurtlar market raflarında bulunabilir olmasına rağmen, geleneksel ev yapımı yoğurt tüketimi hala oldukça yaygındır. Ev yapımı yoğurtlarda toplam laktik asit bakteri sayıları ortalama 1.89x108 kob/g olarak bildirilmiştir. ${ }^{[19]}$

Bu çalışmada birinci basamak standart 3'lü tedaviye (proton pompa inhibitörü (PPI) + klaritromisin + amoksisilin/metronidazol) evde mayalanmış taze yoğurt eklenmesinin Hp eradikasyon hızına ve hasta uyumu üzerine etkisi araştırdık.

\section{Yöntem}

Çalışmaya dahiliye polikliniğine başvuran dispepsi semptomları sebebiyle üst gastrointestinal endoskopisi yapılan 147 hasta alındı. Endoskopik incelemede organik patoloji bulunmayan ve histolojik yöntemle Hp enfeksiyonu teşhis edilen hastalara $\mathrm{Hp}$ eradikasyon tedavisi verildi. Tedavide, tüm hastalara 1 hafta süreyle klaritromisin 500 mg 2x1, amoksisilin 1 gr 2x1, lansoprazol 30 mg 2x1 kullandirıldı. Hastaların evde mayalanmış yoğut tüketimi sorgulandı. Yoğurt tüketenlere tedavi boyunca günlük 2 kase yoğurt tüketmesi önerildi. 14 gün sonunda hastalar ilaç yan etkileri açısından değerlendirildi. Reçete edilen ilaçların \%80'inden fazlasını alan hastalar tedaviye uyumlu kabul edildi.

Tanı ve tedavi stratejisinde kullanılan esas non-invaziv testler üre nefes testi ve monoklonal gaita antijen testleridir. Onaylanmış monoklonal test kullanıldığında dışkıda antijen testi ile üre nefes testi eşit doğruluktadır. ${ }^{[20]}$ Tedavi bitiminden 4 hafta sonra, Hp eradikasyonu gaitada Hp antijen testi ile değerlendirildi.

Daha önce Hp eradikasyon tedavisi alanlar, üst gastrointestinal sistem cerrahisi veya malignite öyküsü olanlar, karaciğer veya böbrek fonksiyon bozukluğu olanlar, pilor darlığı olanlar, gebeler ve penisilin veya diğer antibiyotiklere allerjisi olanlar çalışmaya alınmadı. Ayrıca Hp eradikasyonunu etkileyebileceği için son 4 hafta içinde bizmut tuzları, H2 
histamin reseptör blokerleri, steroid, NSAİ̈, proton pompa inhibitörü, antibiyotik veya probiyotik kullananlar da çalışmaya alınmadı. Çalışma 2004 Helsinki Deklarasyonuna uygun olarak yürütüldü. Tüm hastalar çalışma hakkında bilgilendirildi ve hastalardan bilgilendirilmiş olur alındı. Çalışma yerel etik kurul tarafından onandı.

Tüm hastalar intention-to-treat (ITT) ve per-protocol (PP) analiz ile değerlendirildi. III. Maastricht Konsensus Raporunda Hp enfeksiyonunda İntention-To-Treat (ITT) \%80'in üzerinde eradikasyon oranının olması etkili tedavi olarak kabul edilmiştir. ${ }^{[20]}$

İstatistiksel değerlendirmeler SPSS 10.0 statistical software (SPSS Inc., Chicago, IL,USA) programı ile yapıldı. Sonuçlar kantitatif değişkenler için ortalama \pm standard deviasyon ve kalitatif değerlendirmeler için sayı ve yüzde olarak verildi. Gruplar arasındaki Hp eradikasyon oranları arasındaki fark Ki-kare testi ile değerlendirildi ve $\mathrm{p}<0.05$ anlaml kabul edildi.

\section{Bulgular}

Seksen beşi kadın ve 62'si erkek olmak üzere toplam 147 hasta çalışmaya alındı. Hastaların demografik özellikleri Tablo 1'de verilmiştir. 5 kadın ve 4 erkek hasta hasta tedavi sonrası takiplere gelmedi. 33 hasta ilaci tolere edemedi ve 105 hasta çalışmayı tamamladı (37 erkek, 68 kadın). Çalışma dışı kalan hastaların büyük çoğunluğu yalnız 3’lü tedavi alan gruptand. ITT analizde yoğurt + 3'lü tedavi alan 87 hastanın 50'sinde (\%57.4), sadece 3'lü tedavi alan 60 hastanın 21'inde (\%39) eradikasyon sağlandı. PP analizde yoğurt + 3’lü tedavi alan

\begin{tabular}{|c|c|c|}
\hline & 3'lü tedavi & 3'lü tedavi+yoğurt \\
\hline Hasta sayısı (n, \%) & $40 / 60(66)$ & $65 / 87(74)$ \\
\hline Erkek/kadın (n) & $18 / 22$ & $19 / 36$ \\
\hline Ortalama yaşıSS (yıl) & $46.00 \pm 12.09$ & $46.49 \pm 11.78$ \\
\hline Sigara içen & 10 & 16 \\
\hline Sigara içmeyen & 30 & 49 \\
\hline \multicolumn{3}{|l|}{ Eğitim düzeyi } \\
\hline İlkokul mezunu & 22 & 36 \\
\hline Lise ve dengi okul & 18 & 29 \\
\hline Ek hastalık varlığı & $4 / 40$ & $8 / 65$ \\
\hline \multicolumn{3}{|l|}{ Endoskopi sonucu } \\
\hline Antral gastrit $(n=38)$ & 11 & 27 \\
\hline Duodenal ülser $(n=5)$ & 2 & 3 \\
\hline Eroziv antral gastrit $(n=8)$ & 4 & 4 \\
\hline Nonülser dispepsi (n=7) & 3 & 4 \\
\hline Kronik aktif gastrit $(n=28)$ & 13 & 15 \\
\hline Pangastrit $(n=19)$ & 7 & 12 \\
\hline
\end{tabular}

65 hastanın 50’inde (\%76.9), sadece 3’lü tedavi alan 40 hastanın 21'inde (\%52.5) Hp eradikasyonu sağlandı. Eradikasyon oranları Tablo 1'de verilmiştir. Yoğurt + 3'lü tedavi alanlarda sadece 3'lü tedavi alanlara göre hem ITT hem de PP analizde daha iyi eradikasyon oranları elde edildi. Fark istatistiksel olarak anlamlıyd $(\mathrm{p}=0.009 ; \mathrm{p}=0.028$ ) (Tablo 2).

Tedavi sonrası gaitada antijen testi sonrası 34 hastada Hp (+) saptand. Hp (+) ve Hp (-) gruplar arasında yaş, cinsiyet, sigara kullanımı, eğitim düzeyi açısından anlamlı fark yoktu. Laboratuvar değerlerine bakıldığında $\mathrm{Hp} \mathrm{(+)} \mathrm{olan} \mathrm{grupta} \mathrm{nöt-}$ rofil lenfosit oranı (NLO) istatistiksel olarak anlamlı düzeyde yüksekti ( $\mathrm{p}=0.031$ ) (Tablo 3). Hemoglobin, trombosit, PDW, MPV(mean platelet volume), CRP değerleri arasında istatistiksel fark görülmedi ( $p=0.91 ; p=0.787 ; p=0.078 ; p=0.787$ ).

\begin{tabular}{|c|c|c|c|}
\hline & 3'lü tedavi & 3'lü tedavi+yoğurt & $\mathbf{P}$ \\
\hline \multicolumn{4}{|l|}{ ITT analiz } \\
\hline Eradikasyon hızı & $35 \%(21 / 60)$ & $57.4 \%(50 / 87)$ & $0.009 *$ \\
\hline $95 \% \mathrm{Cl}$ & $28.9-41.1$ & $50.6-64.2$ & \\
\hline \multicolumn{4}{|l|}{ PP analiz } \\
\hline Eradikasyon hızı & $52.5 \%(21 / 40)$ & $76.9 \%(50 / 65)$ & $0.028 *$ \\
\hline $95 \% \mathrm{Cl}$ & $45.7-59.3$ & $70.3-83.5$ & \\
\hline
\end{tabular}

Cl: confidence interval; ITT: intention-to-treat; PP. per-protocol; 3'lü tedavi (klaritromisin $500 \mathrm{mg} 2 \times 1$, amoksisilin 1gr 2x1, lansoprazol 30mg 2x1). *: $p<0.05$; istatistiksel olarak anlamlı fark.

Tablo 3. Eradike olan ve olmayan grupların karşılaştırılması

\begin{tabular}{lccc} 
& $\begin{array}{c}\text { Eradike olan } \\
(\mathbf{n}=\mathbf{7 1})\end{array}$ & $\begin{array}{c}\text { Eradike olmayan } \\
\mathbf{( 3 4 )}\end{array}$ & $\mathbf{p}$ \\
\hline Sayı & 71 & 34 & \\
Yaş & & & \\
$\quad$ Ortalama $\pm S S$ & $46.39 \pm 11.69$ & $46.11 \pm 1234$ & 0.911 \\
Cinsiyet (E/K) & $22 / 49$ & $15 / 19$ & 0.187 \\
Sigara kullanımı (+) & $13 / 26$ & $13 / 26$ & 0.027 * \\
Sigara kullanımı (-) & $58 / 79$ & $21 / 79$ & \\
Eğitim düzeyi & & & \\
$\quad$ Illköğretim & $40 / 58$ & $18 / 58$ & 0.743 \\
$\quad$ Lise ve üstü & $31 / 47$ & $16 / 47$ & \\
Ek hastalık olan & $6 / 12$ & $6 / 12$ & 0.166 \\
Ek hastalık olmayan & $65 / 93$ & $28 / 93$ & \\
Hemoglobin & $12.89 \pm 1.66$ & $12.82 \pm 1.63$ & 0.911 \\
Trombosit (10 $3 / \mathrm{LL})$ & $280.71 \pm 6.35$ & $276.7 \pm 62.64$ & 0,787 \\
PDW (fL) & $15.63 \pm 1.29$ & $15.34 \pm 1.72$ & 0.858 \\
MPV (fL) & $9.84 \pm 1.07$ & $9.50 \pm 0.78$ & 0.078 \\
Nötrofil/lenfosit oranı & $0.56 \pm 0.09$ & $0.60 \pm 0.08$ & 0.031 * \\
Crp (mg/L) & $3.88 \pm 2.51$ & $4.02 \pm 2.48$ & 0.787
\end{tabular}

SS: standart sapma; *: p<0.05; istatistiksel olarak anlamlı fark. 


\section{Tartışma}

HP enfeksiyonu dünyada en sık rastlanılan kronik enfeksiyonlardan biridir ve her yaştan insanı etkilemektedir. Kronik aktif gastritin etyolojik ajanı olan bu mikroorganizmanın peptik ülser, gastrik adenokarsinom ve Maltoma etyolojisinde de rol oynadığı bilinmektedir ve bu nedenlerden dolayı mikroorganizma klas 1 gastrik karsinojen olarak kabul görmekte ve bu bakteriye sahip gastroduodenal ülseri olan hastaların eradikasyon tedavisi alması önerilmektedir

Çalışmamızda birinci son nokta 7 günlük lansoprazol+klaritromisin+amoksisilin tedavi kombinasyonunun Hp eradikasyonu üzerine olan etkisi idi. ITT analizlerine göre $\% 51.4$, PP analizlerine göre \%67.6 idi. Bu veriler ışığında bakıldığında 3’lü tedavi rejimi Hp eradikasyonunda başarılı değildir. Tedavi başarısızlığının en önemli nedeni ilaçlara uyumsuzluk, intolerans ve organizmanın antibiyotik direnci geliştirmesidir. ${ }^{[21]}$ Bizim hastalarımızdan 33 tanesi intolerans nedeni ile tedavi yarım bırakılmış olup, hastalar \%76 oranında tedaviyi tolere etmiştir. İntolerans nedeni ile tedaviyi tamamlayamayan hastaların büyük çoğunluğu klasik tedaviye ek yoğurt almayan gruptandı.

Ulusal bir metaanalizde Türkiye'de Hp eradikasyon oranının PPI-bazlı 3'lü tedavi ile 1997'de \%84 iken, 2004'te \%55.3’e düştüğü belirtilmiştir. ${ }^{[22]}$ Son dönemde ülkemizde yapılan pek çok çalışmada gösterildiği üzere bu durumdan klaritromisin direnci (primer ve/veya sekonder) sorumlu tutulmaktadır. Bununla birlikte yeni antibiyotiklere karşı direnç gelişmesi kaçınılmaz görünmektedir. Ortaya çıan bu durum nedeniyle yeni arayışlar gündeme gelmiştir. Yıllardan beri PPI’lı üçlü tedavilerin etkinliğini arttırmak için birlikte probiyotik kullanımı çalışmaları yapılmaktadır. Yalnız başına probiyotik kullanımının HP enfeksiyonunda eradikasyon sağlayamadığı, yalnızca belli oranlarda supresyon sağladığ1 görülmüştür. PPI ve iki antibiyotikli tedavilerde ise antibiyotiklere bağlı yan etkileri azalttığı saptanmıştır. Probiyotiklerin eradikasyon üzerine etkisi tartışmalıdır; çünkü çelişkili araştırma sonuçları vardır. [23]

Probiyotik içeren yoğurtların tedaviye eklenmesi ile eradikasyon başarısının arttı̆̆ını gösteren çalışmalar olmakla birlikte, ${ }^{[24,25]}$ tam tersini söyleyen çalışmalar da yapılmıştır. ${ }^{[17,26-28]}$

Kim MN ve ark. ${ }^{[17]}$ 3'lü tedaviye probiyotik içeren yoğurt eklemenin HP eradikasyon hızını önemli ölçüde artırdığını göstermiştir (per-protocol (PP) analysis $87.5 \%$ versus $78.7 \%$, $\mathrm{p}=0.037)$.
Bu çalışmada elde ettiğimiz veriler, ev yapımı geleneksel yoğurt ile kombine yapılan üçlü tedavinin HP eradikasyon oranlarını arttırdığını göstermiştir.

İrlanda'da 4742 katılımcıyla yapılan bir araştırmada HP seropozitifliğinin yaşla arttığı, sosyoekonomik düzeyle ters orantılı olduğu raporlanmıştır. ${ }^{[29]}$ Literatüre baktığımızda HP eradikasyon oranının yaşla birlikte arttığını görmekteyiz. ${ }^{[30]} 138$ hasta ile yaptığımız bu çalışmada tedavi sonrası eradikasyon oranı yaştan etkilenmemiştir. Sonuçlardaki farklılık diğer çalışmalarda 18 yaş altı ve 65 yaş üstü fazla sayıda hasta dahil edilmesi ile ilişkili olabilir. Çalışmamızda daha homojen dağılmış bir yaş grubu vardı.

Dünyada yapılan pek çok çalışmaya bakıldığında HP insidansı ile sosyoekonomik düzey ve eğitim düzeyi ile HP enfeksiyonu arasında negatif koorelasyon gözlemlenmektedir. [31, 32] Eğitim seviyesinin artması Hp insidansını azaltmasına rağmen çalışmamızda eradikasyon oranını etkilememiştir. Bununla birlikte istatistiksel olarak anlamlı bir fark olmamasına rağmen ilköğretim seviyesindeki hastalarda erdikasyon oranı daha fazla bulunmuştur.

The American Journal of Medicine'de 2006 yılında yayımlanan 22 çalışmanın metaanalizinde sigaranın eradikasyon başarısını azalttığı raporlanmıştır. ${ }^{[33]} \mathrm{Bu}$ verilerle uyumlu olarak bizim çalışmamızda sigara içmeyen hastalarda eradikasyon oranı daha yüksek bulunmuştur.

İnflamatuvar mediatörler ve Hp ilişkisi son yıllarda merak uyandırmıştır. Farah ve arkadaşları ilk kez nötrofil lenfosit oranı (NLO) ile Hp enfeksiyonu arasında kuvvetli bir ilişkili olduğunu göstermiştir. Başka bir çalışmada Hp (+) olan hastalarIın Hp (-) hastalara göre daha yüksek lökosit, nötrofil ve NLO oranlarına sahip olduğu gösterilmiştir. Bizim çalışmamızda literatür ile uyumlu olarak $\mathrm{Hp}(+)$ hastalarda NLO idi. Fakat CRP, Hp (+) grupta daha yüksek olmasına rağmen aradaki fark istatistiksel olarak anlamlı değildi.

\section{Sonuç}

Sonuç olarak elde ettiğimiz veriler sşığında standart 3'lü tedaviye eklenen geleneksel ev yoğurdu ile Hp eradikasyon hızı artırmıştır. Bununla birlikte, yaş cinsiyet, eğitim düzeyi gibi faktörler eradikasyonu etkilememiştir. Antibiyotik direnci ve tedavi uyumsuzluğu sebebi ile eradikasyonun zorlaştığ $1 \mathrm{Hp}$ enfeksiyonunda tedaviye en azından evde mayalı yoğurt eklemek hem tedavi uyumu, hemde eradikasyonu artırmak için yararlı olabilir. Çalışmamızda hasta sayısı nispeten azdı. Daha geniş hasta popülasyonları üzerinde yapılacak randomize klinik çalışmalara ihtiyaç vardır. 


\section{Açıklamalar}

Etik Kurul Onayı: Sağlık Bilimleri Üniversitesi Okmeydanı Eğitim ve Araştırma Hastanesi Klinik Araştırmalar Etik Kurulunun 11/06/2018 tarihli toplantısında 1332 nolu kararla etik kurul onayı almıştır.

Hakem Değerlendirmesi: Dış bağımsız.

Çıkar Çatışması: Bildirilmemiştir.

Yazarlık Katkıları: Konsept - Ş.A.Y.; Dizayn - G.Y.; Denetim G.Y.; Meteryal - Ş.A.Y.; Veri toplama veya işleme - Ş.A.Y.; Analiz ve yorumlama - Ş.A.Y.; Literatür arama - Ş.A.Y.; Yazan - Ş.A.Y.; Kritik revizyon - G.Y.

\section{Kaynaklar}

1. Uemura N, Okamoto S, Yamamoto S, Matsumura N, Yamaguchi S, Yamakido M, et al. Helicobacter pylori infection and the development of gastric cancer. N Engl J Med 200113;345:784-9.

2. Wotherspoon AC. A critical review of the effect of Helicobacter pylori eradication on gastric MALTlymphoma. Curr Gastroenterol Rep 2000;2:494-8.

3. Siavoshi F, Malekzadeh R, Daneshmand M, Smoot DT, Ashktorab H. Association between Helicobacter pylori Infection in gastric cancer, ulcers and gastritis in Iranian patients. Helicobacter 2004;9:470.

4. Komoto K, Haruma K, Kamada T, Tanaka S, Yoshihara M, Sumii K, et al. Helicobacter pylori infection and gastric neoplasia: correlations with histological gastritis and tumor histology. Am J Gastroenterol 1998;93:1271-6.

5. Mihara M, Haruma K, Kamada T, Komoto K, Yoshihara M, Sumii $\mathrm{K}$, et al. The role of endoscopic findings for the diagnosis of Helicobacter pyloriinfection: evaluation in a country with high prevalence of atrophic gastritis. Helicobacter 1999;4:40-8.

6. McColl KE. Clinical practice. Helicobacter pylori infection. $\mathrm{N}$ Engl J Med 2010;362:1597-604.

7. Megraud F, Coenen S, Versporten A, Kist M, Lopez-Brea M, Hirschl AM, et al. Helicobacter pylori resistance to antibiotics in Europe and its relationship to antibiotic consumption. Gut 2013;62:34-42.

8. Tong JL, Ran ZH, Shen J, Zhang CX, Xiao SD. Meta-analysis: the effect of supplementation with probiotics on eradicationrates and adverse events during Helicobacter pylori eradication therapy. Aliment Pharmacol Ther 2007;25:155-68.

9. Lesbros-Pantoflickova D, Corthésy-Theulaz I, Blum AL. Helicobacter pylori and probiotics. J Nutr 2007;137:812S-8S.

10. Sachdeva A, Nagpal J. Effect of fermented milk-based probiotic preparations on Helicobacter pylorieradication: a systematic review and meta-analysis of randomized-controlledtrials. Eur J Gastroenterol Hepatol 2009;21:45-53.

11. Zou J, Dong J, Yu X. Meta-analysis: Lactobacillus containing quadruple therapy versus standardtriple first-line therapy for Helicobacter pylori eradication. Helicobacter 2009;14:97-107.

12. Lourens-Hattingh A, Viljoen BC. Yogurt as probiotic carrier food. International Dairy Journal 2001;11:1-17.

13. Akpinar A, Yerlikaya O, Kiliç S. Antimicrobial activity and antibiotic resistance of Lactobacillus delbrueckii ssp bulgaricus and Streptococcus thermophilus strain isolated from Turkish homemade yoghurts. African journal of microbiology research 2011;5:675-82

14. Petti S, Tarsitani G, Simonetti D'Arca A. Antibacterial activity of yoghurt against viridans streptococci in vitro. Arch Oral Biol 2008;53:985-90.

15. Kabir AM, Aiba Y, Takagi A, Kamiya S, Miwa T, Koga Y. Prevention of Helicobacter pylori infection by lactobacilli in a gnotobioticmurine model. Gut 1997;41:49-55.

16. Aiba Y, Suzuki N, Kabir AM, Takagi A, Koga Y. Lactic acid-mediated suppression of Helicobacter pylori by the oraladministration of Lactobacillus salivarius as a probiotic in a gnotobiotic murinemodel. Am J Gastroenterol 1998;93:2097-101.

17. Kim MN, Kim N, Lee SH, Park YS, Hwang JH, Kim JW, et al. The effects of probiotics on PPI-triple therapy for Helicobacter pylorieradication. Helicobacter 2008;13:261-8.

18. Sakamoto I, Igarashi M, Kimura K, Takagi A, Miwa T, Koga Y. Suppressive effect of Lactobacillus gasseri OLL 2716 (LG21) on Helicobacter pylori infection in humans. J Antimicrob Chemother 2001;47:709-10.

19. Durak Y, Keleş F, Uysal A, Aladağ MO. Konya yöresi ev yapımı yoğurtların mikrobiyolojik özelliklerinin araştırılması S.Ü. Ziraat Fakültesi Dergisi 2008;22:113-117.

20. Malfertheiner P, Megraud F, O'Morain C, Bazzoli F, El-Omar E, Graham D, et al. Current concepts in the management of Helicobacter pylori infection: the Maastricht III Consensus Report. Gut 2007;56:772-81.

21. Osato MS, Reddy R, Reddy SG, Penland RL, Malaty HM, Graham DY. Pattern of primary resistance of Helicobacter pylori to metronidazole or clarithromycin in the United States. Arch Intern Med 2001;161:1217-20.

22. Alkim $\mathrm{H}$, Iscan $\mathrm{M}, \mathrm{Oz} \mathrm{F}$. Effectiveness of ranitidine bismuth citrate and proton pump inhibitor basedtriple therapies of Helicobacter pylori in Turkey. Libyan J Med 2011;6.

23. Navarro-Rodriguez T1, Silva FM, Barbuti RC, Mattar R, Moraes-Filho JP, de Oliveira MN, et al. Association of a probiotic to a Helicobacter pylori eradication regimen does not increase efficacy or decreases the adverse effects of the treatment: a prospective, randomized, double-blind, placebo-controlled study. BMC Gastroenterol 2013;13:56.

24. Huebner ES, Surawicz CM. Probiotics in the prevention and treatment of gastrointestinal infections. Gastroenterol Clin North Am 2006;35:355-65.

25. Ornelas IJ, Galvan-Potrillo M, López-Carrillo L. Protective effect of yoghurt consumption on Helicobacter pylori seropositivityin a Mexican population. Public Health Nutr 2007;10:1283-7.

26. Armuzzi A, Cremonini F, Bartolozzi F, Canducci F, Candelli M, Ojetti V, et al. The effect of oral administration of Lactobacillus GG on antibiotic-associatedgastrointestinal side-effects during Helicobacter pylori eradication therapy. Aliment Pharmacol Ther 2001;15:163-9.

27. Cremonini F, Di Caro S, Covino M, Armuzzi A, Gabrielli M, Santarelli L, et al. Effect of different probiotic preparations on anti-helicobacter pylori therapy-related side effects: a parallel group, triple blind, placebo-controlled study. Am J Gastroenterol 2002;97:2744-9.

28. Tursi A, Brandimarte G, Giorgetti GM, Modeo ME. Effect of Lactobacillus casei supplementation on the effectiveness and tolerability of a new second-line 10-day quadruple therapy after 
failure of a firstattempt to cure Helicobacter pylori infection. Med Sci Monit 2004;10:CR662-6.

29. Murray LJ, McCrum EE, Evans AE, Bamford KB. Epidemiology of Helicobacter pylori infection among 4742 randomly selectedsubjects from Northern Ireland. Int J Epidemiol 1997;26:880-7.

30. Labenz J, Leverkus F, Börsch G. Omeprazole plus amoxicillin for cure of Helicobacter pylori infection. Factorsinfluencing the treatment success: Scand J Gastroenterol 1994;29:1070-5.

31. Celiński K, Kurzeja-Mirosław A, Słomka M, Cichoz-Lach H, Madro A, Kasztelan-Szczerbińska B. The effects of environ- mental factors on the prevalence of Helicobacter pyloriinfection in inhabitants of Lublin Province. Ann Agric Environ Med 2006;13:185-91.

32. Brown LM, Thomas TL, Ma JL, Chang YS, You WC, Liu WD, et al. Helicobacter pylori infection in rural China: demographic, lifestyle and environmental factors. Int J Epidemiol 2002;31:638-45. 33. Suzuki T, Matsuo K, Ito H, Sawaki A, Hirose K, Wakai K, et al. Smoking increases the treatment failure for Helicobacter pylori eradication. Am J Med 2006;119:217-24. 